\title{
Overexpression of CYP3A5 attenuates inducibility and activity of CYP3A4 in HepG2 cells
}

\author{
ZEMIN KUANG ${ }^{1}$, ZHIJUN HUANG $^{2,3}$, YING LI $^{2,3}$, GUOPING YANG $^{2,3}$, MEILIN LIU ${ }^{4}$ and HONG YUAN ${ }^{2,3}$ \\ ${ }^{1}$ Department of Hypertension, Beijing Anzhen Hospital of Capital Medical University, Beijing 100029; \\ ${ }^{2}$ Center of Clinical Pharmacology, The Third Xiangya Hospital, Central South University; \\ ${ }^{3}$ Human Research Center of Hypertension, Changsha, Hunan 410013; ${ }^{4}$ Department of \\ Geriatrics, The First Hospital, Peking University, Beijing 100034, P.R. China
}

Received December 9, 2013; Accepted October 1, 2014

DOI: $10.3892 / \mathrm{mmr} .2014 .3022$

\begin{abstract}
There have been conflicting reports regarding the catalytic role of cytochrome $\mathrm{P} 450$ (CYP)3A5, which range from deeming it irrelevant to suggesting it is equally as important as CYP3A4, the most potent and abundant catalytic cytochrome enzyme in the human liver. This was partially attributed to the fact that CYP3A5 is highly polymorphic. However the importance of other underlying mechanisms remain unclear. The aim of the present study was to investigate the interaction between these enzymes. A human HepG2 hepatocellular line stably overexpressing CYP3A5 was constructed. The results suggested that CYP3A5 does not affect CYP3A4 expression directly. However, overexpression of CYP3A5 attenuated the inducibility of CYP3A4 in response to dexamethasone. A luciferase reporter assay indicated that this attenuation was due to a decrease in CYP3A4 promoter activity. Furthermore, a pharmacokinetic assay using quinidine and amlodipine showed that CYP3A4 enzyme activity per mg of microsomal protein was also decreased in the group overexpressing CYP3A5 compared with the dexamethasone-induced control group. In conclusion, the current study demonstrated that CYP3A5 may affect CYP3A4 at the transcriptional level and may thus modify CYP3A4 expression and activity in the presence of substrates and inducers. The results indicate that CYPs may interact with each other under certain conditions and that this interaction may be a novel mechanism by which drug-drug interactions are mediated.
\end{abstract}

Correspondence to: Professor Hong Yuan, Center of Clinical Pharmacology, The Third Xiangya Hospital, Central South University, 138 Tongzipo Road, Changsha, Hunan 410013, P.R. China E-mail: yuanhong01@vip.sina.com

Key words: CYP3A4, CYP3A5, dexamethasone, inducibility, pharmacokinetic

\section{Introduction}

The human cytochrome P450 (CYP) enzyme system is a superfamily of paralogs that are dominantly expressed in the adult liver and gastrointestinal tract (1). They catalyze phase-I metabolism of a wide variety of exogenous chemicals, resulting in substrates that are more water soluble, and thus facilitating excretion or further transformation into nontoxic compounds (2).

CYP3A4 is the predominant P450 enzyme expressed in human liver, and was understood to metabolize $>60 \%$ of clinically prescribed drugs (3). CYP3A4 is not detectable prior to birth, but its expression gradually increases thereafter (4). Despite the heterogeneity of liver CYP3A4 expression among adult humans, CYP3A4 expression may also be affected transiently by xenobiotics, mostly its substrate, including CYP inducers, such as rifampin, phenobarbital, clotrimazole and dexamethasone (5), and inhibitors, such as verapamil, erythromycin, nifedipine, testosterone, midazolam and amiodarone (6). Changes in intestinal and liver CYP3A4 activity may have a significant effect on drug metabolism and thereby affect bioavailability of these compounds (7). Thus, increasing attention has been focused on the involvement of CYP3A4 in drug-drug interactions (DDI) $(8,9)$.

The CYP3A5 gene is located on chromosome 7q22.1, upstream of CYP3A4, and shares $84 \%$ similarity in amino acid sequence with CYP3A4 (10). CYP3A4 and 5 catalyze similar and overlapping metabolic reactions, including nifedipine oxidation, testosterone $6 \beta$-hydroxylation, erythromycin $\mathrm{N}$-demethylation, cyclosporine oxidation and hydroxylation of benzodiazepine, midazolam, triazolam, alprazolam and terfenadine (11). Although it may differ in catalytic activity and regioselectivity, some reports have argued that CYP3A5 is more important than CYP3A4 for overall drug clearance $(12,13)$ and also in the pathogenesis of certain diseases, such as hypertension (14). It exhibited comparable or greater metabolic activity than CYP3A4 for certain substrates, including carbamazepine (12). Unfortunately, the majority of reported studies have not distinguished the activity of CYP3A4 from CYP3A5, and use CYP3A4 to reflect the activity of either enzyme. However, there are important differences between the enzymes. CYP3A5 is highly-expressed 
in the adult kidney (15) rather than the liver, in contrast to CYP3A4. In addition expression of CYP3A5 in human liver varies among individuals and species from being undetectable to comprising $>50 \%$ of total CYPs present in this organ (16). Therefore the importance of CYP3A5 in metabolism may have been underestimated.

A number of studies have emphasized the polymorphism of CYP3A5 and have investigated the relevance of this to clinical issues in treating hypertension (17), and during liver (18) and kidney $(19,20)$ transplantation. However, conflicting results have been obtained. For example, a study examining the effect of CYP3A5 deactivating mutations, reported no association between expression of this enzyme and the response of blood pressure to amlodipine among African-Americans (21) By contrast, CYP3A5 expression had a significant impact on the response of blood pressure to amlodipine in healthy Korean subjects (22). In order to resolve this conflicting information, the present study investigated whether external factors are involved in modulating the expression of CYP3A5 in response to certain drugs. The effect of overexpression of CYP3A5 on the ability of dexamethasone (DEX) to induce CYP3A4 activity was investigated. The effect of CYP3A5 on the promoter activity of CYP3A4 in the presence of DEX was also examined. Finally the metabolism of a representative substrate drug, amlodipine in response to CYP3A5 overexpression was investigated.

\section{Materials and methods}

Chemicals and plasmid construction. Lipofectamine ${ }^{\circledR} 2000$ (18324-012) was obtained from Invitrogen Life Technologies, (Carlsbad, CA, USA). The protein G-Sepharose (17-0618-01) was obtained from GE Healthcare Biosciences (Pittsburgh, PA, USA).. Monoclonal mouse anti-human CYP3A4 (sc-53850) and CYP3A5 antibodies (sc-53616) were obtained from Santa Cruz Biotechnology, Inc. (Dallas, TX, USA). Monoclonal mouse anti- $\alpha$-tubulin antibody (T5168), quinidine and amlodipine were purchased from Sigma-Aldrich (St Louis, MO, USA). Horseradish peroxidase-conjugated anti-mouse secondary antibody was obtained from GE Healthcare Biosciences. Other chemicals used to prepare buffers were obtained from Shenggong Ltd. (Shanghai, China). Dexamethasone was reconstituted in dimethyl sulfoxide (DMSO) at concentration of $1 \mathrm{mM}$.

The GFP-CYP3A5 plasmid was constructed by in-frame ligation of a CYP3A5 coding sequence amplified from HepG2 cDNA with pIRES2-eGFP (Clonetech Laboratories, Mountain View, CA, USA). pGL3-CYP3A4-promoter consisted of a pGL3-basic vector (Promega Corporation, Madison, WI, USA) inserted with the 5'-flanking region of CYP3A4 (from $-1.6 \mathrm{~kb}$ to $+100 \mathrm{bp}$ to the start codon).

Cell culture and stable transfection of CYP3A5. Human HepG2 cells (Institute of Biochemistry and Cell Biology, Shanghai Institutes for Biological Sciences, Chinese Academy of Sciences, Shanghai, China) were cultured from a nitrogen preserved batch and maintained with Dulbecco's modified Eagle's medium containing $10 \%$ fetal bovine serum, $100 \mathrm{U} / \mathrm{ml}$ penicillin, and $0.1 \mathrm{mg} / \mathrm{ml}$ streptomycin in
Table I. Sequences of primers and siRNAs.

\begin{tabular}{ll}
\hline Primer and siRNA & \multicolumn{1}{c}{ Primer/siRNA sequences } \\
\hline CYP3A4 promoter & F: gttcacaggaagcagcacaaa \\
R: gagagccatcactactttccttact \\
CYP3A5 & F: attcagcaagaagaacaaggaca \\
overexpression & R: tggtgttctcaggcacagat \\
CYP3A4 RT & F: ccttacatatacacaccctttggaag \\
& R: ggttgaagaagtcctcctaagct \\
CYP3A5 RT & F: aggcgggaagcagagaaag \\
& R: ggggtcttgtggattgttgag \\
CYP3A5 siRNA & Si-A: tgcctttgttgggaaatgttttg \\
& Si-B: tccattatttctctcaataatac \\
& Si-C: gagttattctaaggatttctact
\end{tabular}

siRNA, small interfering RNA; F, forward; R, reverse; RT, reverse transcription.

a humidified incubator at $37^{\circ} \mathrm{C}$ with $5 \% \mathrm{CO}_{2}$. Cells $\left(5 \times 10^{5}\right)$ were seeded onto a $6-\mathrm{cm}$ dish and transfected with $10 \mu \mathrm{g}$ purified GFP-CYP3A5 plasmid at $70 \%$ confluence using Lipofectamine 2000 according to the manufacturer's instructions. Cells were then reseeded onto three $10-\mathrm{cm}$ dishes with complete medium supplemented with $500 \mu \mathrm{g} / \mathrm{ml}$ of G418 (Cellgro, Manassas, VA, USA) for colony selection. Clones were selected 2 weeks post-transfection and expanded for RNA and protein verification. Positive CYP3A5 ${ }^{+}$HepG2 clones were frozen for subsequent experiments.

RNA extraction and reverse transcription-quantitative polymerase chain reaction $(R T-q P C R)$. HepG2 cells were seeded in 6 -well plates at a density of $5 \times 10^{5}$ cells per well Following indicated treatments, samples were washed with phosphate-buffered saline (PBS) and lysed with $1 \mathrm{ml}$ TRIzol ${ }^{\circledR}$ (Invitrogen Life Technologies). RNA extractions were performed according to the manufacturer's instructions. Total RNA (1 $\mu \mathrm{g})$ from each sample was used for cDNA synthesize using a Revert Aid First Strand cDNA Synthesis kit (Thermo Fisher Scientific, Waltham, MA, USA). qPCR was then performed using All-in-One SYBR ${ }^{\circledR}$ Green qPCR mix (GeneCopoeia, Rockville, MD, USA) on an Applied Biosystem 7300 qPCR module. The sequences of CYP3A4/5 detecting primers are provided in Table I, and human GAPDH (23) was used as internal control. The qPCR conditions were set as follows: Pre-heating at $95^{\circ} \mathrm{C}$ for $10 \mathrm{~min}$, followed by 40 cycles of $95^{\circ} \mathrm{C}$ for $15 \mathrm{sec}$ and $60^{\circ} \mathrm{C}$ for $15 \mathrm{sec}$, and a final melting curve stage. Fold changes in mRNA levels were calculated using the $2^{-\Delta \Delta C t}$ method.

Protein and western blotting. Treated HepG2 cells were washed twice with PBS and lysed in radioimmunoprecipitation assay buffer (50 mm Tris, pH 7.5; $150 \mathrm{~mm} \mathrm{NaCl} ; 0.1 \%$ SDS, $0.5 \%$ sodium deoxycholate; $1 \%$ Triton X-100; and $1 \mathrm{~mm}$ phenylmethanesulfonyl fluoride). Cell lysates were then sonicated on ice for $30 \mathrm{sec}$ and cell debris was removed by centrifugation at $10,000 \mathrm{x} \mathrm{g}$ for $15 \mathrm{~min}$. The protein concentrations of each 
sample were determined by a bicinchoninic acid assay (Pierce Biotechnology, Inc., Rockford, IL, USA) according to the manufacturer's instructions. Total protein $(30 \mu \mathrm{g})$ was loaded onto $10 \%$ SDS-PAGE gels, and transferred to polyvinylidene fluoride membranes (GE Healthcare Biosciences). Each membrane was blocked with $5 \%$ non-fat milk and incubated with the indicated antibodies. The membranes were incubated with primary antibodies at room temperature for $4 \mathrm{~h}$ or at $4^{\circ} \mathrm{C}$ overnight and horseradish peroxidase-conjugated anti-mouse secondary antibody $(1: 5,000)$ at room temperature for $1 \mathrm{~h}$. Signals were visualized using Enhanced Chemiluminescence (ECL) western blotting substrate (Thermo Fisher Scientific). Monoclonal mouse anti-human CYP3A4/5 antibodies were diluted at a ratio of 1:1,000 in Tris-buffered saline containing $5 \%$ bovine serum albumin and $0.1 \%$ sodium azide. . Each blot was stripped using Stripping Buffer $(0.5 \mathrm{mM} \mathrm{NaCl} / 0.2 \mathrm{mM}$ acetic acid) and reprobed. Monoclonal mouse anti- $\alpha$-tubulin $(1: 3,000)$ was used as an internal control.

Luciferase reporter assay. Luciferase reporter assays were performed using a Dual-Luciferase ${ }^{\circledR}$ Reporter Assay system (Promega Corporation). Specifically, pGL3-CYP3A4-promotor Luciferase reporter vectors were co-transfected with or without CYP3A5 siRNA, as indicated, using Lipofectamine 2000 in 24 -well plates, where HepG2 cells were at $70 \%$ confluence. Following treatment, cells were washed and lysed with $80 \mu 1$ lysis buffer from the Dual-Luciferase ${ }^{\circledR}$ Reporter kit. Following three freeze and thaw cycles, cell lysates were centrifuged at $4^{\circ} \mathrm{C}$ and $9,300 \mathrm{x}$ g for $10 \mathrm{~min}$ and $10 \mu \mathrm{l}$ was mixed with $100 \mu \mathrm{l}$ of buffer LARII by pipetting in luminometer tubes. Firefly fluorescence was then read on a Turner DesignsTD-20/20 luminometer (Promega Corporation) immediately. The fluorescence of Renilla luciferase was measured in a second reading following the addition of $100 \mu \mathrm{l}$ Stop \& Glo ${ }^{\circledR}$ Reagent and vortexed for $5 \mathrm{sec}$ at $\sim 100 \mathrm{rpm}$.

High performance liquid chromatography-mass spectometry (HPLC-MS) detection of CYP3A4 substrate metabolites. Enzyme catalytic activity experiments were performed using microsomes extracted from treated HepG2 cells, as previously described (24). Microsomes were suspended in PBS $(0.1 \mathrm{mM}, \mathrm{pH} 7.4)$. Microsomal protein $(50 \mu \mathrm{g})$ in $250 \mu \mathrm{l}$ total incubation volume was achieved in all samples following addition of substrates. Samples were pre-incubated for $5 \mathrm{~min}$ in a $37^{\circ} \mathrm{C}$ shaker, and enzyme reactions were initiated by applying nicotinamide adenine dinucleotide phosphate (NADPH) regenerating buffer to a working concentration of $1 \mathrm{mM} \mathrm{NADP}{ }^{+}, 7.5 \mathrm{mM}$ isocitric acid, $10 \mathrm{mM}$ magnesium chloride and 0.2 units of isocitric dehydrogenase. Total organic solvent did not exceed $1 \% \mathrm{v} / \mathrm{v}$. The linear range of each substrate was determined. Quinidine was chosen as a selective probe for CYP3A4 and amlodipine, widely used clinically as an antihypertensive, was used as a representative CYP3A4-metabolized drug. A starting concentration of $1 \mathrm{mM}$ quinidine and amlodipine was used for the $20 \mathrm{~min}$ incubation. Reactions were terminated with $100 \mu 1$ acetonitrile containing $0.1 \mu \mathrm{M}$ tolbutamide as an internal standard. Samples were then analyzed by HPLC- MS, as previously described (25).
Statistical analysis. The data were collected by at least three independent experiments. Averages and standard deviations were calculated using GraphPad Prism version 5.0 (GraphPad Software, Inc., La Jolla, CA, USA) software. Student's t test was used for evaluating differences and a $\mathrm{P}<0.05$ was considered to indicate a statistically significant difference.

\section{Results}

Overexpression of CYP3A5 does not affect the expression of CYP3A4. Increasing evidence has demonstrated that CYP3A5 is important in the pathogenesis of hypertension (14), not only via the control of water retention $(26,27)$, but also through the metabolism of certain antihypertensive drugs. However, conflicting data have been reported regarding the influence of CYP3A5 on the response of blood pressure to amlodipine $(21,28)$. It was hypothesized that there may be additional factors involved in moderating the metabolism of drugs other than the innate catalytic ability of CYP3A5.

The effect of CYP3A5 on CYP3A4 expression was investigated in a HepG2 cell line, which was induced to overexpress CYP3A5 using GFP-CYP3A5. The expression level of CYP3A5 was confirmed by western blotting and RT-qPCR. As shown in Fig. 1, RNA and protein levels of CYP3A4 were not affected by CYP3A5 overexpression, suggesting that CYP3A4 is not direct regulated by CYP3A5.

Overexpression of CYP3A5 reduces induction of CYP3A4 expression by DEX. The effect of clinical conditions, such as the presence of substrates or inducers was then investigated. DEX was selected due to its wide range of clinical applications. HepG2-wild type (WT) and HepG2-CYP3A5 ${ }^{+}$cells were seeded onto 6 -well plates at $5 \times 10^{5}$ cells/well. Following overnight adhesion, $100 \mathrm{nM}$ DEX was applied for a further $48 \mathrm{~h}$ incubation. Cells were then harvested for RNA and protein analysis. As hypothesized, CYP3A4 RNA as well as protein levels were significantly elevated following DEX induction in the WT groups compared with the control group (Fig. 2). However, this induction was suppressed in the cells overexpressing CYP3A5. This indicates that CYP3A5 affects CYP3A4 indirectly under certain conditions.

Overexpression of CYP3A5 attenuates CYP3A4 promoter activity in the presence of $D E X$. To further explore the effect of overexpression of CYP3A5 on DEX induction of CYP3A4 expression, the 5'-flanking region of CYP3A4 was cloned as described. Three short interfering RNAs (siRNAs) were designed and synthesized at GenePharma Ltd. (Shanghai, China), and transfected into HepG2 cells. The effects of silencing CYP3A5 were examined with western blotting and the most potent siRNA ( $\mathrm{Si}-\mathrm{C})$ was selected for subsequent experiments (Fig. 3). HepG2-WT and HepG2-CYP3A5 $5^{+}$cells were seeded onto 24 -well plates at a density of $4 \times 10^{4}$ cells/well. The purified pGL3-CYP3A4-promoter plasmid was co-transfected with $\mathrm{Si}-\mathrm{C}$ or control RNA following cell adhesion. Fresh medium containing $100 \mathrm{nM}$ DEX was exchanged at $6 \mathrm{~h}$ post-transfection. Samples were harvested, and Luciferase activity was measured, following $48 \mathrm{~h}$ incubation as described. In concordance with the CYP3A4 expression data shown above, the CYP3A4 promoter activity was unaffected by 
A

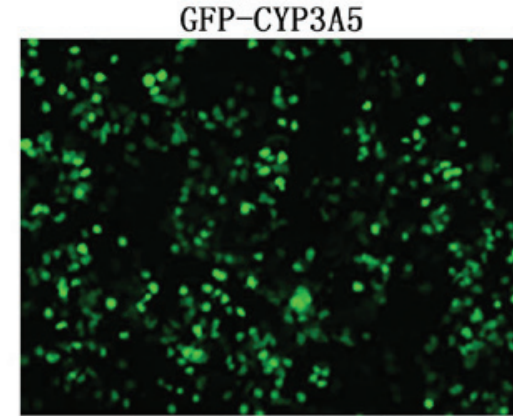

C

Magnification $10 \mathrm{X}$

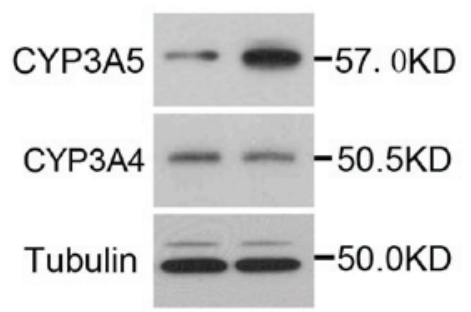

B

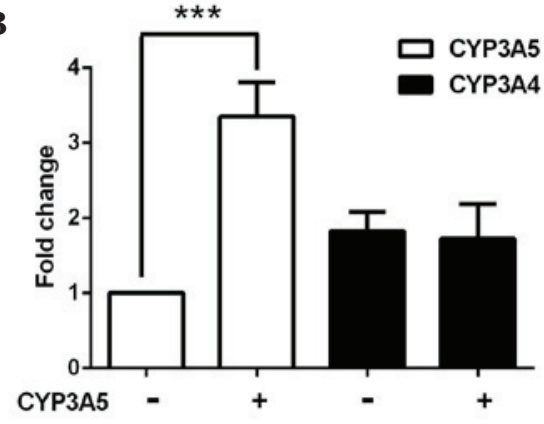

D

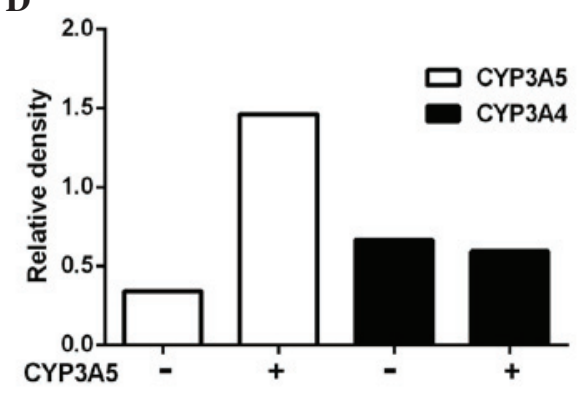

Figure 1. Effect of overexpression of CYP3A5 on the expression of CYP3A4. (A) A HepG2 cell strain stably overexpressing CYP3A5 was constructed. (B) mRNA levels of CYP3A4/5 of WT and CYP3A $5^{+} \mathrm{HepG} 2$ cells were measured using reverse transcription-quantitative polymerase chain reaction. Data are presented as the mean \pm standard deviation $(n=3) .{ }^{* * *} \mathrm{P}<0.001$. (C) Protein analysis of CYP3A4/5 of WT and CYP3A $5^{+}$HepG2 cells using western blotting. (D) Density quantitation of western blot signal shown in panel C. CYP, cytochrome P450 (subsequent characters denote family, subfamily and polypeptide); WT, wild type.

A

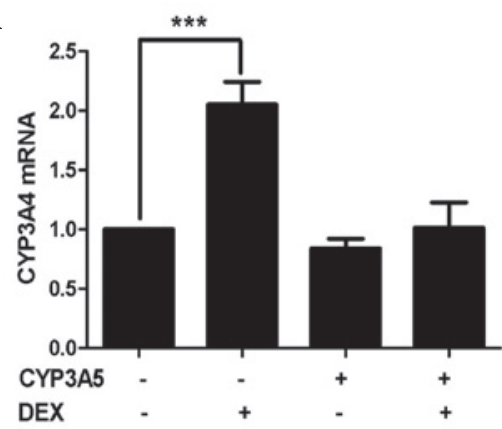

B

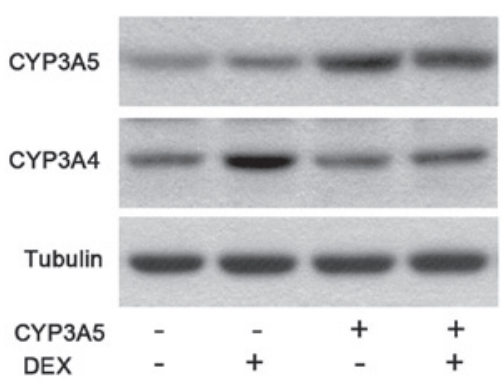

Figure 2. Effect of overexpression of CYP3A5 on DEX-induced CYP3A4 expression. (A) Reverse transcription-quantitative polymerase chain reaction detection of CYP3A4 mRNA levels in WT or CYP3A5 ${ }^{+}$HepG2 cells treated with or without DEX. Data are presented as the mean \pm standard deviation $(\mathrm{n}=3)$. ${ }^{* * * *} \mathrm{P}<0.001$. (B) Protein expression of CYP3A4/5 following DEX application on WT or CYP3A5 ${ }^{+}$HepG2 cells. CYP, cytochrome P450 enzyme (subsequent characters denote family, subfamily and polypeptide); DEX, dexamethasone; WT, wild type.

overexpressing or silencing of CYP3A5. DEX was shown to stimulate activation of the CYP3A4 promoter, which was also consistent with previous reports. Notably, overexpression of CYP3A5 suppressed CYP3A4 promoter activity compared with control in the presence of DEX. This suggests that an abundance of CYP3A5 may affect CYP3A4 expression at the transcriptional level under certain conditions.

Overexpression of CYP3A5 decreases the activity of CYP3A4 in the presence of DEX. To further investigate the impact of the suppression of CYP3A4 function in the presence of DEX, CYP3A4 activity in cells that had undergone the same treatment was measured. In order to remove the influence of CYP3A5, quinidine was used as a selective CYP3A4 probe based on the availability of clinical DDI data and the structural characteristics of the probe substrate (20). The 3-hydroxylated quinidine generated in each group was normalized to non-treated controls, in order to assess the alteration in CYP3A4 activity. As in the luciferase experiments, the enzyme activity was also reduced compared with DEX-treated controls (Fig. 4A). Greater activity was observed in the CYP3A5-silenced group. CYP3A4 levels appeared to be inversely associated with the expression of CYP3A5 in the presence of DEX.

The impact of this interaction on potential clinical events was examined using amlodipine, a calcium channel blocker, widely used as an antihypertensive drug. The un-transformed amlodipine was measured by HPLC-MS/MS and normalized to the levels in the DEX-untreated control (Fig. 4B). The rate of amlodipine metabolism increased in cells overexpressing CYP3A5, although it was not statistically significant. The 
A

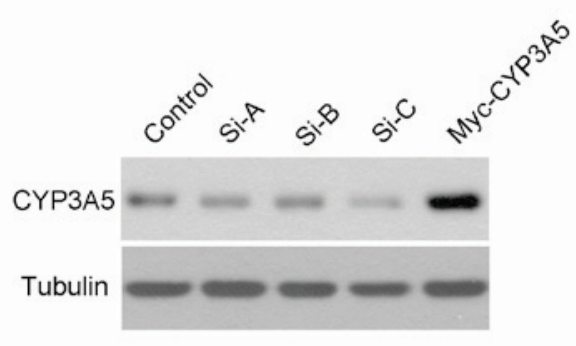

B

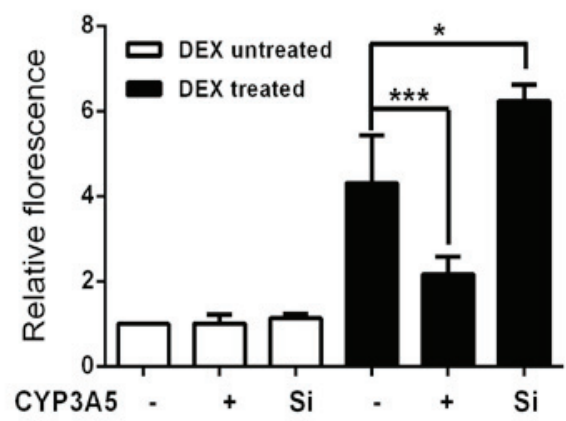

Figure 3. Effect of overexpression of CYP3A5 on CYP3A4 promoter activity in the presence of DEX. (A) Potency of CYP3A5 siRNAs were evaluated by WB. Myc-CYP3A5 was used as transfection control. (B) Promoter activity of CYP3A4 was detected by a luciferase activity assay. -, WT HepG2 cells; +, CYP3A5 ${ }^{+}$-overexpressing cells; $\mathrm{Si}, \mathrm{CYP} 3 \mathrm{~A} 5$ silenced cells. Data are presented as the mean \pm standard deviation $\left(\mathrm{n}=3\right.$ ). ${ }^{*} \mathrm{P}<0.05$ and ${ }^{* * * *} \mathrm{P}<0.001$. CYP, cytochrome P450 enzyme (subsequent characters denote family, subfamily and polypeptide); DEX, dexamethasone; siRNA, small interfering RNA; WT, wild type.

A

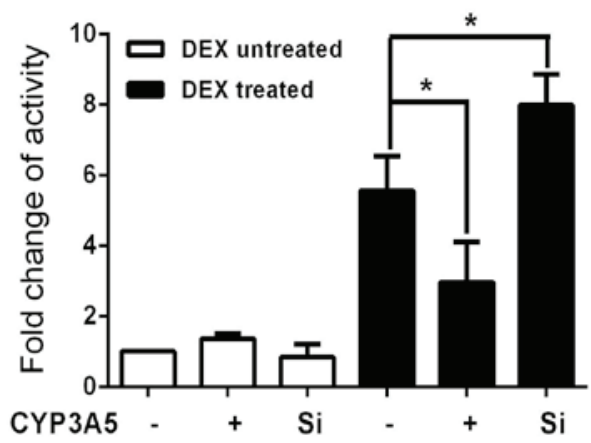

B

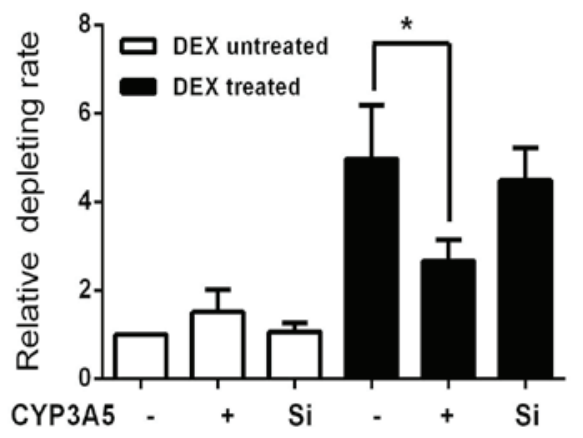

Figure 4. Effect of overexpression of CYP3A5 on CYP3A4 enzyme activity following DEX application. (A) CYP3A4 activity of HepG2 microsome detected by quinidine. (B) Amlodipine metabolic dynamics. All data were normalized to the first column. -, WT HepG2 cells; +, CYP3A $5^{+}$-overexpressing cells; $\mathrm{Si}, \mathrm{CYP} 3 \mathrm{~A} 5$ silenced cells. Data are presented as the mean \pm standard deviation $(\mathrm{n}=5)$. $\mathrm{P}<0.05$. CYP, cytrochrome $\mathrm{P} 450$ enzyme (subsequent characters denote family, subfamily and polypeptide); DEX, dexamethasone; WT, wild type.

clearance of amlodipine increased following DEX induction, and this induction was reduced by CYP3A5 overexpression. This may be attributed to the fact that the metabolism of amlodipine by CYP3A4 is four times higher than that of CYP3A5. Following DEX induction, the rate of metabolism of amlodipine in the CYP3A5-silenced group was not significantly changed compared with the DEX-treated control group. This result indicates that CYP3A4 is the primary enzyme involved in the metabolism of amlodipine. However, CYP3A5 may influence CYP3A4 metabolism of this and other substrates following exposure to DEX.

\section{Discussion}

Primary hepatocytes are a valuable in vitro model with which to identify compounds that are potentially toxic to humans. However, there are a number of disadvantages to the use of primary hepatocytes, including a shortage of available human liver material, limited proliferation ability and loss of metabolic activity after a limited number of passages, which have constrained its application. A number of groups have explored the possibility of obtaining hepatocytes from differentiated adult or embryonic stem cells, or immortalized human hepatocytes, but this technique remains inconvenient. HepG2 cells express the predominant liver functional CYP isoforms associated with drug metabolism. CYP3A7 is the dominant isoform in these cells compared with CYP3A4 in the human adult liver. Thus, HepG2 cells possess a phenotype most similar to that of the fetal liver $(29,30)$. Although less suitable for predicting effects on metabolism in humans, HepG2 cells provide a system that is easy to handle and reproducible, and thus suitable for the investigation of gene regulation. Quinidine was reported to be predominantly metabolized by CYP3A4 (25), which is consistent with the observation in the present study that overexpression of CYP3A5 did not increase the rate of quinidine metabolism significantly in the absence of other compounds. The results confirm that quinidine is a reliable marker of CYP3A4 activity.

Environmental factors, such as cigarette smoking and food intake induce the expression of CYPs and increase clearance of phenacetin and theophylline (31). Hepatic diseases, such as hepatitis B virus infection and cirrhosis, and age, gender, hormones, inflammation and pregnancy alter the expression pattern of CYP enzymes (32). The complexity of the transcriptional regulation of CYP3A4 has been attributed to the response of CYP3A4 to such factors. Transcription factors, such as 
pregnane X receptor (PXR; -362/+53) (33), constitutive androstane receptor (CAR) (34), nuclear factor I (-243/-220) (29), differentially expressed in chondrocyte 1 (35) and hepatocyte nuclear factor $4 \mathrm{a}(\mathrm{HNF} 4 \mathrm{a})(36)$ have been reported to account for a component of CYP3A4 inter-individual variability. Constitutive liver enhancer module of CYP3A4 (CLEM4) (37) and CCAAT-enhancer-binding proteins (C/EBP) response elements (38) were also found within its proximal promoter. However a significant degree of CYP3A4 variation remains unexplained. Epigenetic regulation of CYP3A4 has also been explored recently. The $12 \mathrm{~kb}$ CYP3A4 regulatory region shows highly variable $\mathrm{CpG}$ methylation in the adult liver, which corresponds to important CYP3A4 transcription factor binding sites, including xenobiotic responsive enhancer module, CLEM4, C/EBP and HNF4a (39). In addition, a high degree of methylation was observed in the fetal liver, which is consistent with the minimal expression of CYP3A4 at this stage. The results from the promoter activity experiments demonstrated that CYP3A5 does not affect CYP3A4 transcription directly. However, in the presence of DEX, the promoter activity appears to be inversely correlated with the expression of CYP3A5 (Fig. 3). This data may indicate that excessive CYP3A5 prevents DEX from binding to its response elements. However, which transcriptional factors are involved remains to be elucidated.

CYP3A5 protein expression was found to be highly variable in a manner that was generally independent of age but dependent on race (4). The expression of the CYP3A5*3 polymorphism results in a truncated mRNA (40). This polymorphism is observed at a similar frequency in Chinese and Japanese populations, but three times higher in Caucasian populations $(41,42)$, which implies that more Asian subjects are extensive CYP3A5 metabolizers. CYP3A5 expression appears to be inducible via the glucocorticoid receptor, PXR and CAR- $\beta$, as is the case for CYP3A4. However, the 5'-flanking regions of CYP3A5 shares only $60 \%$ sequence similarity with that of CYP3A4. The low homology may be one of the factors that differentiates their regulation (43). This may explain the different effects of DEX on the induction of the expression of each of these genes (Fig. 2). CYP3A5 has been associated with disease, however these associations are independent to its drug metabolizing function. CYP3A 5*1 homozygotes may have higher systolic blood pressure (14). Certain combined CYP3A4/CYP3A5 haplotypes exhibit differential susceptibility to prostate cancer (44). Females positive for CYP3A $5^{*} 1$ appear to reach puberty earlier, which may affect their risk of developing breast cancer (45). The results from the current study demonstrating an interaction between CYP3A5 and CYP3A4 extends the potential impact of CYP3A5 polymorphisms and variations in expression, since the metabolic capacity of CYP3A4 appears to be higher than of CYP3A5 and CYP3A7 (46) for the majority of substrates.

DEX is a potent synthetic member of the glucocorticoid class of steroid drugs that are widely used as anti-inflammatory and immunosuppressant treatments. DEX is preferentially metabolized by CYP3A4 into $6 \beta-\mathrm{OH}-\mathrm{DEX}$ in the human adult liver. Thus DEX has been used in a number of studies as a probe for CYP3A4 activity. However, DEX is also a potent inducer of CYP3A4 (47). Evidence reported by Pascussi et al (48) demonstrated that the mechanism underlying DEX induction of CYP3A4 is concentration-related, as a low dose (10-100 nM) of DEX induced CYP3A4 via the glucocorticoid receptor, whilst a high concentration $(10 \mu \mathrm{M})$ activates CYP3A4 through the PXR pathway. In the present study, DEX strongly induced CYP3A4 in HepG2 cells, whilst the induction by CYP3A5 was limited (Fig. 2). This is consistent with previous reports $(30,46)$. Since DEX is also a common substrate of CYP3A5, although at a relatively lower metabolic rate, it is postulated that the overexpression of CYP3A5 accelerates the metabolism of DEX, and thus reduces the level of expression of CYP3A4 that it can induce. Thus, DEX may be a bridge linking CYP3A4 and CYP3A5 function. A similar observation was made that DEX increased erythromycin breath test (ERBT) only in CYP3A 5*1 non-carriers as they may be more susceptible to the inductive effects of DEX due to lower basal CYP3A activity (49).

Adverse drug interactions are an important cause of morbidity, hospitalization, and mortality. Drug interactions are be the fourth leading cause of death in hospitalized patients in the US (50). The greatest risk of drug interactions occurs due to effects on the cytochrome system. CYP3A4, the most prevalent cytochrome P450, accounts for $30-50 \%$ of drugs metabolized by type I enzymes. Previous DDI studies have focused on drug metabolism by single specific enzymes, such as CYP3A4 or CYP3A5. However, conflicting results have been reported using this model. For instance, CYP3A $5 * 3$ carriers require a lower dose of substrate drugs, such as cyclosporine and tacrolimus (51). However, CYP3A5 3 showed no association with the response of blood pressure to amlodipine in African-Americans with early hypertensive renal disease (21). The current study demonstrated that the contribution of CYP3A5 may be an important source of inter-individual variability in response to drugs. Furthermore, the identification of this novel interaction may provide further insights when predicting drug metabolism and designing individualized treatment regimes, particularly when a patient with multiple co-morbidities is prescribed more than two drugs.

\section{Acknowledgements}

This study was supported by the National Basic Research Program of China (grant no. 2011CB512001), The National Key Technology R\&D Program (grant no. 2012BAI37B05), the National Natural Science Foundation of China (grant no. 81273594) and the Fundamental Research Funds for the Central Universities of Central South University (grant no. 2012zzts036).

\section{References}

1. Kruijtzer CM, Beijnen JH and Schellens JH: Improvement of oral drug treatment by temporary inhibition of drug transporters and/or cytochrome P450 in the gastrointestinal tract and liver: an overview. Oncologist 7: 516-530, 2002.

2. Casarett LJ and Doull J (eds): Toxicology: The Basic Science of Poisons. Macmillan Publishing Company, London, UK, 1975.

3. Anzenbacher P and Anzenbacherová E: Cytochromes P450 and metabolism of xenobiotics. Cell Mol Life Sci 58: 737-747, 2001.

4. Stevens JC, Hines RN, Gu C, et al: Developmental expression of the major human hepatic CYP3A enzymes. J Pharmacol Exp Ther 307 : 573-582, 2003.

5. Luo G, Cunningham M, Kim S, et al: CYP3A4 induction by drugs: correlation between a pregnane $\mathrm{X}$ receptor reporter gene assay and CYP3A4 expression in human hepatocytes. Drug Metab Dispos 30: 795-804, 2002. 
6. Katoh M, Nakajima M, Yamazaki H and Yokoi T: Inhibitory effects of CYP3A4 substrates and their metabolites on P-glycoprotein-mediated transport. Eur J Pharm Sci 12: 505-513, 2001.

7. Kivistö KT, Niemi $M$ and Fromm MF: Functional interaction of intestinal CYP3A4 and P-glycoprotein. Fundam Clin Pharmacol 18: 621-626, 2004.

8. Galetin A, Burt H, Gibbons L and Houston JB: Prediction of time-dependent CYP3A4 drug-drug interactions: impact of enzyme degradation, parallel elimination pathways, and intestinal inhibition. Drug Metab Dispos 34: 166-175, 2006.

9. Wang RW, Newton DJ, Liu N, Atkins WM and Lu AY: Human cytochrome P-450 3A4: in vitro drug-drug interaction patterns are substrate-dependent. Drug Metab Dispos 28: 360-366, 2000.

10. Walsky RL, Obach RS, Hyland R, et al: Selective mechanism-based inactivation of CYP3A4 by CYP3cide (PF-04981517) and its utility as an in vitro tool for delineating the relative roles of CYP3A4 versus CYP3A5 in the metabolism of drugs. Drug Metab Dispos 40: 1686-1697, 2012

11. Yuan R, Madani S, Wei XX, Reynolds K and Huang SM: Evaluation of cytochrome P450 probe substrates commonly used by the pharmaceutical industry to study in vitro drug interactions. Drug Metab Dispos 30: 1311-1319, 2002.

12. Huang W, Lin YS, McConn DJ II, et al: Evidence of significant contribution from CYP3A5 to hepatic drug metabolism. Drug Metab Dispos 32: 1434-1445, 2004.

13. Dai Y, Hebert MF, Isoherranen N, et al: Effect of CYP3A5 polymorphism on tacrolimus metabolic clearance in vitro. Drug Metab Dispos 34: 836-847, 2006

14. Kivistö KT, Niemi M, Schaeffeler E, et al: CYP3A5 genotype is associated with diagnosis of hypertension in elderly patients: data from DEBATE study. Am J Pharmacogenomics 5: 191-195, 2005.

15. Dai Y, Iwanaga K, Lin YS, et al: In vitro metabolism of cyclosporine A by human kidney CYP3A5. Biochem Pharmacol 68 : $1889-1902,2004$

16. Kuehl P, Zhang J, Lin Y, et al: Sequence diversity in CYP3A promoters and characterization of the genetic basis of polymorphic CYP3A5 expression. Nat Genet 27: 383-391, 2001.

17. Zhang YP, Zuo XC, Huang ZJ, et al: CYP3A5 polymorphism, amlodipine and hypertension, J Hum Hypertens 28: 145-149, 2013

18. Shi Y, Li Y, Tang J, et al: Influence of CYP3A4, CYP3A5 and MDR-1 polymorphisms on tacrolimus pharmacokinetics and early renal dysfunction in liver transplant recipients. Gene 512: 226-231, 2013.

19. Zuo XC, Ng CM, Barrett JS, et al: Effects of CYP3A4 and CYP3A5 polymorphisms on tacrolimus pharmacokinetics in Chinese adult renal transplant recipients: a population pharmacokinetic analysis Pharmacogenet Genomics 23: 251-261, 2013.

20. Suzuki Y, Itoh H, Fujioka T, et al: Association of plasma concentration of $4 \beta$-hydroxycholesterol with CYP3A5 polymorphism and plasma concentration of indoxyl sulfate in stable kidney transplant recipients. Drug Metab Dispos 42: 105-110, 2014.

21. Bhatnagar V, Garcia EP, O'Connor DT, et al: CYP3A4 and CYP3A5 polymorphisms and blood pressure response to amlodipine among African-American men and women with early hypertensive renal disease. Am J Nephrol 31: 95-103, 2010.

22. Kim KA, Park PW, Lee OJ, et al: Effect of CYP3A 5*3 genotype on the pharmacokinetics and pharmacodynamics of amlodipine in healthy Korean subjects. Clin Pharmacol Ther 80: 646-656, 2006.

23. Overbergh L, Valckx D, Waer M and Mathieu C: Quantification of murine cytokine mRNAs using real time quantitative reverse transcriptase PCR. Cytokine 11: 305-312, 1999.

24. Paine MF, Khalighi M, Fisher JM, et al: Characterization of interintestinal and intraintestinal variations in human CYP3A-dependent metabolism. J Pharmacol Exp Ther 283 $1552-1562,1997$.

25. Galetin A, Brown C, Hallifax D, Ito K and Houston JB: Utility of recombinant enzyme kinetics in prediction of human clearance: impact of variability, CYP3A5, and CYP2C19 on CYP3A4 probe substrates. Drug Metab Dispos 32: 1411-1420, 2004.

26. Ghosh SS, Basu AK, Ghosh S, et al: Renal and hepatic family $3 \mathrm{~A}$ cytochromes P450 (CYP3A) in spontaneously hypertensive rats. Biochem Pharmacol 50: 49-54, 1995.

27. Zhang L, Miyaki K, Wang W and Muramatsu M: CYP3A5 polymorphism and sensitivity of blood pressure to dietary salt in Japanese men. J Hum Hypertens 24: 345-350, 2010.

28. Ho H, Pinto A, Hall SD, et al: Association between the CYP3A5 genotype and blood pressure. Hypertension 45: 294-298, 2005.
29. Riffel AK, Schuenemann E and Vyhlidal CA: Regulation of the CYP3A4 and CYP3A7 promoters by members of the nuclear factor I transcription factor family. Mol Pharmacol 76: 1104-1114, 2009.

30. Maruyama M, Matsunaga T, Harada E and Ohmori S: Comparison of basal gene expression and induction of CYP3As in HepG2 and human fetal liver cells. Biol Pharm Bull 30 2091-2097, 2007.

31. Sarkar MA and Jackson BJ: Theophylline N-demethylations as probes for P4501A1 and P4501A2. Drug Metab Dispos 22: 827-834, 1994

32. Badyal DK and Dadhich AP: Cytochrome P450 and drug interactions. Ind J Pharmacol 33: 248-259, 2001.

33. Moore LB, Parks DJ, Jones SA, et al: Orphan nuclear receptors constitutive androstane receptor and pregnane $\mathrm{X}$ receptor share xenobiotic and steroid ligands. J Biol Chem 275: 15122-15127, 2000.

34. Drocourt L, Ourlin JC, Pascussi JM, Maurel P and Vilarem MJ: Expression of CYP3A4, CYP2B6, and CYP2C9 is regulated by the vitamin $\mathrm{D}$ receptor pathway in primary human hepatocytes. J Biol Chem 277: 25125-25132, 2002.

35. Mao Z, Luan X, Cao G, et al: DEC1 binding to the proximal promoter of CYP3A4 ascribes to the downregulation of CYP3A4 expression by IL-6 in primary human hepatocytes. Biochem Pharmacol 84: 701-711, 2012.

36. Tirona RG, Lee W, Leake BF, et al: The orphan nuclear receptor HNF4alpha determines PXR-and CAR-mediated xenobiotic induction of CYP3A4. Nat Med 9: 220-224, 2003.

37. Matsumura K, Saito T, Takahashi Y, et al: Identification of a novel polymorphic enhancer of the human CYP3A4 gene. Mol Pharmacol 65: 326-334, 2004.

38. Jover R, Bort R, Gómez-Lechón MJ and Castell JV: Down-regulation of human CYP3A4 by the inflammatory signal interleukin-6: molecular mechanism and transcription factors involved. FASEB J 16: 1799-1801, 2002.

39. Kacevska M, Ivanov M, Wyss A, et al: DNA methylation dynamics in the hepatic CYP3A4 gene promoter. Biochimie 94: 2338-2344, 2012.

40. Busi F and Cresteil T: CYP3A5 mRNA degradation by nonsense mediated mRNA decay. Mol Pharmacol 68, 808-815, 2005.

41. Hu YF, He J, Chen GL, et al: CYP3A5*3 and CYP3A4*18 single nucleotide polymorphisms in a Chinese population. Clin Chim Acta 353: 187-192, 2005.

42. Chou FC, Tzeng SJ and Huang JD: Genetic polymorphism of cytochrome P450 3A5 in Chinese. Drug Metab Dispos 29: 1205-1209, 2001.

43. Jounaïdi Y, Guzelian PS, Maurel P and Vilarem MJ: Sequence of the 5'-flanking region of CYP3A5: comparative analysis with CYP3A4 and CYP3A7. Biochem Biophys Res Commun 205: $1741-1747,1994$

44. Zeigler-Johnson C, Friebel T, Walker AH, et al: CYP3A4, CYP3A5, and CYP3A43 genotypes and haplotypes in the etiology and severity of prostate cancer. Cancer Res 64: 8461-8467, 2004.

45. Bajpai P, Tripathi AK and Agrawal D: Genetic polymorphism of CYP3A5 in Indian chronic myeloid leukemia patients. Mol Cell Biochem 336: 49-54, 2010.

46. Krusekopf S, Roots I and Kleeberg U: Differential drug-induced mRNA expression of human CYP3A4 compared to CYP3A5, CYP3A7 and CYP3A43. Eur J Pharmacol 466: 7-12, 2003

47. $\mathrm{Lu} \mathrm{C}$ and Li AP: Species comparison in P450 induction: effects of dexamethasone, omeprazole, and rifampin on P450 isoforms $1 \mathrm{~A}$ and $3 \mathrm{~A}$ in primary cultured hepatocytes from man, Sprague-Dawley rat, minipig, and beagle dog. Chem Biol Interact 134: 271-281, 2001.

48. Pascussi JM, Drocourt L, Gerbal-Chaloin S, et al: Dual effect of dexamethasone on CYP3A4 gene expression in human hepatocytes. Sequential role of glucocorticoid receptor and pregnane X receptor. Eur J Biochem 268: 6346-6358, 2001.

49. Roberts PJ, Rollins KD, Kashuba AD, et al: The influence of CYP3A5 genotype on dexamethasone induction of CYP3A activity in African Americans. Drug Metab Dispos 36: 1465-1469, 2008

50. Pirmohamed M, James S, Meakin S, et al: Adverse drug reactions as cause of admission to hospital: prospective analysis of 18,820 patients. BMJ 329: 15-19, 2004.

51. Zhao Y, Song M, Guan D, et al: Genetic polymorphisms of CYP3A5 genes and concentration of the cyclosporine and tacrolimus. Transplant Proc 37: 178-181, 2005. 